\title{
Intravenous infusions of ifosfamide/mesna and perturbation of warfarin anticoagulant control
}

\author{
Graeme Hall ${ }^{3}$, Michael J. Lind ${ }^{1}$, Moses Huang ${ }^{2}$, Anthony Moore'2, \\ Alan Gane ${ }^{2}$, J. Trevor Roberts ${ }^{2}$ and Brian M.J. Cantwell ${ }^{1}$ \\ University Department of ${ }^{1}$ Clinical Oncology, Departments of ${ }^{2}$ Radiotherapy and ${ }^{3}$ Pharmacy, \\ Newcastle General Hospital, Westgate Road, Newcastle upon Tyne NE4 6BE, UK.
}

\begin{abstract}
Summary: We report three patients who had serious disturbance of anticoagulant control while receiving ifosfamide/mesna-containing cytotoxic chemotherapy in association with warfarin. In view of the likely biological potentiation of warfarin by ifosfamide/mesna, close monitoring of anticoagulant control should be performed during the co-administration of these drugs.
\end{abstract}

\section{Introduction}

Patients with cancer receiving cytotoxic chemotherapy may suffer from thromboembolic complications that require anticoagulant drugs. ${ }^{1,2}$ Ifosfamide is an oxazaphosphorine alkylating agent that is increasingly being used with the uroprotector mesna to treat a variety of cancers. We have recently observed three patients to have serious disturbance of the international normalized ratio (INR), a measure of anticoagulant control, while receiving treatment with ifosfamide/mesnacontaining combination cytotoxic chemotherapy for cancer. In our institution patients who are not receiving anticoagulant therapy should have an INR of 1.0 and the target range for appropriate anticoagulation using warfarin is an INR of 2-4.

\section{Case reports}

A 16 year old girl with a malignant ovarian endodermal sinus tumour was given warfarin for a left femoral vein thrombosis and her INR was stable while she received an oral dose of warfarin $4 \mathrm{mg}$ each day for 2 months. She was given etoposide $100 \mathrm{mg} / \mathrm{m}^{2}$ in 1 litre of normal saline which was infused intravenously over 4 hours. Cisplatin $20 \mathrm{mg} / \mathrm{m}^{2}$ in 1 litre of normal saline was then infused intravenously over 6 hours. Ifosfamide plus mesna both in doses of $3 \mathrm{~g} / \mathrm{m}^{2}$ dissolved in 3 litres of dextrose saline were infused intravenously, each litre over a time period of 8 hours. Forty-eight hours after initiation of intravenous chemotherapy the INR had risen to 8.4. Oral

Correspondence: B.M.J. Cantwell, M.D., M.R.C.P., M.R.C.P.I.

Accepted: 25 April 1990 medications started just prior to intravenous chemotherapy were nabilone $2 \mathrm{mg}$ /day domperidone $120 \mathrm{mg} /$ day and lorazepam $1 \mathrm{mg} /$ day.

A 61 year old woman with advanced breast cancer was given warfarin for an axillary vein thrombosis and anticoagulation control was stable on an oral dose of warfarin $4 \mathrm{mg}$ daily and the INR was 2.3. Intravenous chemotherapy was given with doxorubicin $40 \mathrm{mg} / \mathrm{m}^{2}$ as an intravenous bolus and ifosfamide $5 \mathrm{~g} / \mathrm{m}^{2}$ was given as a 24 hour infusion in 3 litres of normal saline, preceded by $1 \mathrm{~g} / \mathrm{m}^{2}$ intravenous bolus of mesna. Four $\mathrm{g} / \mathrm{m}^{2}$ of mesna were given by intravenous infusion over a 32 hour period starting at the same time as ifosfamide. When given together ifosfamide and mesna were mixed in the same infusion fluid. Forty-eight hours after initiation of chemotherapy the INR had risen to 7.5. The INR subsequently fell to 1.3 on cessation of warfarin, but 5 days after chemotherapy the INR remained below 2.0 despite oral doses of warfarin of $10 \mathrm{mg}$ daily. Five days later the INR was within the therapeutic range and stable on a maintenance warfarin dose of $4 \mathrm{mg}$ per day. Oral medications started just before intravenous chemotherapy were domperidone $120 \mathrm{mg}$ /day, lorazepam $2 \mathrm{mg}$ /day and dexamethasone $8 \mathrm{mg} /$ day.

A 25 year old man was given warfarin following partial resection of a retroperitoneal malignancy. Anticoagulation control was stable on $\mathbf{4 m g}$ of warfarin each day with an INR of 2.1. Intravenous chemotherapy was given and consisted of doxorubicin $20 \mathrm{mg} / \mathrm{m}^{2}$ and vincristine $2 \mathrm{mg}$ both given as intravenous bolus injections. Ifosfamide, a total of $9 \mathrm{~g} / \mathrm{m}^{2}$ and mesna $10.5 \mathrm{~g} / \mathrm{m}^{2}$ dissolved in 9 litres of normal saline were given by intravenous infusions, each litre infused over an 8-hour period. Forty-eight hours after initiation of chemotherapy the INR was noted to be 5.5 and warfarin was 
discontinued, but it was possible to reintroduce warfarin 2 days later with stabilization of the INR on a dose of $4 \mathrm{mg}$ of warfarin each day. Oral anti-emetic therapy given just prior to chemotherapy consisted of intermittent metoclopramide $10 \mathrm{mg}$, intermittent chlorpromazine $25 \mathrm{mg}$ and the patient was also taking intermittent diamorphine in a dose of 5-10 mg. Three weeks later the patient was given a second course of combination chemotherapy. Immediately prior to this the INR was 2.8 and rose again to $4.8,3$ days after initiation of the second course of chemotherapy.

Two other patients receiving ifosfamide/mesnacontaining chemotherapy but not given warfarin had blood tests to examine the effect of this chemotherapy on the INR. Both patients had preand post-treatment INR values of 1.0 .

\section{Discussion}

Ifosfamide is an analogue of cyclophosphamide with similar but not identical biological characteristics. ${ }^{3}$ Although cyclophosphamide itself is only $12-24 \%$ protein bound, its metabolites are more widely bound to protein (nor-nitrogen mustard $67 \%$, phosphoramide mustard $33 \%){ }^{4}$ It is likely

\section{References}

1. Cantwell, B.M.J., Carmichael, J., Ghani, S.E. \& Harris, A.L Thromboses and thromboemboli in patients with lymphoma during cytotoxic chemotherapy. Br Med J 1988, 297: 179- 180.

2. Cantwell, B.M.J., Mannix, K.A., Roberts, J.T., Ghani, S.E. \& Harris, A.L. Thromboembolic events during combination chemotherapy for germ cell malignancy. Lancet 1988, ii: 1086-1087.

3. Zalupski, M. \& Baker, L.H. Ifosfamide. J Natl Cancer Inst 1988, 80: 556-566.

4. Jardine, F.E., Feneslau, C., Appler, M., Kar, M.N., Brundrett, R. \& Colvin, M. Quantification by gas chromatographychemical ionisation mass-spectrometry of cyclophosphamide, phosphoramide mustard and nor-nitrogen mustard in the plasma and urine of patients receiving cyclophosphamide therapy. Cancer Res 1978, 38: 408-415. that the protein binding of ifosfamide and its metabolites follows a similar pattern. The increase in INR could thus be due to an increase in free warfarin levels due to displacement from protein binding by ifosfamide metabolites.

A second mechanism may be the inhibition of cytochrome $\mathbf{P}_{450}$ mediated metabolism of warfarin by ifosfamide or one of its metabolites. Cyclophosphamide has been shown to inhibit microsomal enzyme function in the rat. ${ }^{5}$ Ifosfamide has been shown to auto-induce its own metabolism, presumably due to induction of cytochrome $\mathrm{P}_{450}{ }^{6}$ Ifosfamide could therefore inhibit initially the enzymes metabolizing warfarin but then induce them and this could explain the relative warfarin resistance observed in our second patient who initially had potentiation of warfarin effect.

A possible effect of mesna or anti-emetics or the other cytotoxic drugs used in combination in causing pertubation of anticoagulant control cannot be excluded. However, treatment with ifosfamide/mesna was common to all 3 cases and was a likely cause of the observed potentiation of anticoagulant effect. Patients who are given warfarin and are concomitantly prescribed ifosfamide should have close monitoring of anticoagulant control, even if this has previously been stable.

5. Donelli, M.G., Franchi, G. \& Ross, R. The effect of cytotoxic agents on drug metabolism. Eur J Cancer 1970, 6: 125-126. 6. Lind, M.J., Margison, J.M., Cerny, T., Thatcher, N. \& Wilkinson, P.M. Comparative pharmacokinetics and alkylating activity of fractionated intravenous and oral ifosfamide in patients with bronchogenic carcinoma. Cancer Res 1989, 49: 753-757. 